\title{
Besearch S Sulure \\ Bacteria and Antimicrobial Resistance in Children at Damascus Hospital
}

Fares Kahal ( $\sim$ Fareskahal@hotmail.com )

Syrian Private University

Ahmed Helwani

Syrian Private University

Maher Al Bani MD

Syrian Private University

\section{Research Article}

Keywords: Paediatric, Antibiotic resistance, Sensitivity, Syria

Posted Date: January 28th, 2022

DOI: https://doi.org/10.21203/rs.3.rs-1301053/v2

License: (c) (1) This work is licensed under a Creative Commons Attribution 4.0 International License.

Read Full License 


\section{Abstract}

Background: Antimicrobial resistance is the third top-ranking global causative of death.

Aims: The aim is to evaluate antimicrobial resistance and sensitivity to aid the assessment of the efficacy in Syrian management protocols.

Methods: A retrospective study was conducted to analyze children with positive culture results presenting with nosocomial or community-acquired infections in Damascus Hospital, Damascus, Syria.

Results: 117 patients with 183 positive culture results were included in the study. The most antibioticresistant bacterium was Pseudomonas aeruginosa. The highest bacterial antibiotic resistance occurred with Ceftriaxone, Pseudomonas aeruginosa (89\%), Escherichia coli (77\%), Enterobacter (74\%), and Proteus (42\%). Staphylococcus sensitivity towards vancomycin was only $3 \%$. The mortality was 19(16\%), nosocomial infection $11(55 \%)$ and community infections $9(45 \%)$.

Conclusion: The efficacy of the remaining antibiotics to which the bacteria are sensitive must be maintained through rationalization of its use. Strict precautionary measures must be implemented to ban the illegal dispensation of antibiotics.

\section{Introduction}

The third top-ranking worldwide cause of death is due to antibiotic resistance. Antibiotic resistance is highly related to both high mortality rates and high medical costs in the healthcare system. New predictions show there were 4.95 million deaths in 2019 due to bacterial antibiotic resistance. Projections revealed that death will soar to an estimated 10 million deaths by 2050 as a result of antibiotic resistance. ${ }^{[1]}$

In Syria, self-diagnosing and self-medicating is a common ritual among Syrians, seeking advice from local pharmacists is customary to avoid doctor visit expenses with a prevalence of $57 \% .{ }^{[2,3]}$ Over-thecounter sales of antibiotics have been reported in various countries of the Middle East; the prevalence of antibiotic self-medication ranged between 19 and $82 \% .{ }^{[4]} \mathrm{A}$ study carried out on pharmacists in the capital, Damascus, found that $87 \%$ of pharmacists sold antibiotics without prescription, $10 \%$ sold with prescription, and $3 \%$ refused to sell antibiotics without prescription; ${ }^{[5]}$ despite the law issued by the Ministry of Health in 1988 that specified that antibiotics were not among the drugs that could be sold without a prescription. ${ }^{[6]}$ This has resulted in the emergence of antimicrobial resistance in both nosocomial and community infections in Syria. Also, with the increased number of Syrian immigrants, this local problem evolved into a global issue as several studies conducted in Europe on Syrian immigrants have revealed many multidrug-resistant pathogens (MDR) bacteria. The presence of MDR gram-negative microbes was found in $60 \%$ of the immigrants who underwent the microbiological tests. ${ }^{[7}$, 8] 
This study aims to provide an insight into the Syrian paediatric population at Damascus hospital during the current Syrian crisis and the Covid-19 pandemic. This study aims to describe the epidemiology, culture results, bacterial antibiotic resistance and sensitivity, and outcomes of children with positive culture results presenting with nosocomial or community-acquired infections. Evaluating antimicrobial resistance and sensitivity is an integral part of assessing efficacy in our management protocols.

\section{Methods}

A cross-sectional descriptive retrospective study was conducted at the Pediatric Division in Damascus Hospital (Al Mujtahid Hospital), Damascus, Syria, between 7/31/2020 and 7/31/2021. 117 patients with 183 positive culture results were included in the study. The inclusion criteria for evaluation were medical records of children admitted to the pediatric division $\leq 13$ with positive culture results. Cases with positive culture results attending the pediatric accident and emergency department were excluded (70 cases). Of 123 records found, 6 patients were admitted with positive culture results on 2 separate occasions. Data extracted from medical records in the pediatric archive included: gender, age, diagnosis, outcome (recovery or death), culture result, type of infection acquired (community or nosocomial), and types of antibiotics administered before and after culture results. Culture reports included information on the type of sample (Urine, blood, sputum, cerebral spinal fluid, cutaneous swabs, lower respiratory tract secretions, respiratory tract swabs, ascites fluid, pleural fluid, pus, swabs from central and venous catheters, swabs from urinary catheter, swabs from intubation tubes, and swabs from ICU ventilators), bacteria cultured, and bacteria's sensitivity and resistance towards antibiotics. When culture reports were missing from medical records in the pediatric archive, the culture report was found among the archives of the histopathology laboratory. Ethical approval was obtained from Damascus Hospital Institutional Review Board (IRB). Data frequencies, percentages, and means were calculated via SPSS (Statistical package for social sciences) 25.0 program for Windows.

\section{Results}

Of 117 patients, 55(47\%) were male, and 62(53\%) were females, the ages ranged from 3 hours to 13 years. Cases of positive culture results peaked at the age of 0-11 months 59(50\%) were children aged 13 years $(1 \%)$ represented a minority (Figure 1 ).

The bacteria that appeared in the samples were: Staphylococcus (33\%), Enterobacter (21\%), Pseudomonas aeruginosa (15\%), Escherichia Coli (12\%), Proteus (10\%), Klebsiella (3\%), Acinetobacter (2\%), Streptococcus (2\%), Gram-positive bacilli (1\%), Serratia (1\%), and coliform (0.5\%). The percentage of nosocomial infections was $35 \%$, gram-positive bacteria accounted for $34 \%$ of the bacteria in the sample, while gram-negative bacteria constituted $66 \%$ of the sample. Among the community-acquired bacteria, the percentage of gram-positive bacteria was $37 \%$, and the percentage of gram-negative was $63 \%$. Among the nosocomial bacteria, the percentage of gram-positive bacteria was $33 \%$, and the percentage of gram-negative bacteria was $67 \%$. Staphylococcus aureus was the most prevalent bacteria in both nosocomial and community-acquired infections. 
Staphylococcus was the most common bacteria among all age groups. The distribution of bacteria differed across each age group: Neonatal stage (< 4weeks): Staphylococcus (55\%), Enterobacter (22\%), Escherichia coli (11\%), and Proteus (11\%). Infant stage (1month-1year): Staphylococcus aureus (33\%), Enterobacter (23\%), Escherichia coli (15\%), Proteus (8\%), and Pseudomonas aeruginosa (8\%). Early childhood stage (1-6years): Staphylococcus (26\%), Pseudomonas aeruginosa (22\%), Enterobacter (18\%), Proteus : (13\%), and Escherichia coli (9\%). Late childhood stage (6-13years): Staphylococcus aureus (35\%), Pseudomonas aeruginosa (21\%), Enterobacter (21\%), Escherichia coli (10\%), and Proteus (6\%).

The most common foci of infections were the urinary tract (37\%), blood (25\%), respiratory tract (21\%), cutaneous (6\%), and meninges (3\%). The most common nosocomial infections were blood (31\%), and urinary tract $(31 \%)$. They were followed by respiratory tract $(17 \%)$, cutaneous $(9 \%)$, and meninges $(6 \%)$. The most common community-acquired infections were the urinary tract $(42 \%)$, and respiratory tract (24\%). They were followed by blood (21\%), cutaneous (4\%), and meninges (3\%).

The most cultured bacteria found to cause urinary tract infections were Enterobacter (30\%), Staphylococci (22\%), and Escherichia coli (20\%), followed by Proteus (14\%), Pseudomonas (10\%), Klebsiella (2\%), Coliform (2\%). The most cultured bacteria in respiratory infections were Staphylococcus (30\%), Pseudomonas aeruginosa (23\%), and Enterobacter (19\%), followed by Klebsiella (8\%), Escherichia coli (8\%), Serratia (8\%), and Proteus (4\%). Regarding cerebrospinal fluid (CSF), staphylococcus was the most common causative (75\%), followed by streptococci (12.5\%) and gram-positive bacilli (12.5\%). Regarding skin infections, staphylococcus was the most common causative (58\%), followed by Proteus (17.\%), Pseudomonas aeruginosa (8\%), Enterobacter (8\%), and Escherichia coli (8\%). Regarding sepsis, the most common microorganisms were staphylococcus (48\%), followed by Pseudomonas aeruginosa (7\%), Acinetobacter (7\%), Escherichia coli (4\%), Klebsiella (4\%), Proteus (4\%), and gram-positive bacilli $(2 \%)$.

The following 3 antibiotics were used as pretreatments: Ceftriaxone $67 \%$, and was appropriate in $50 \%$ of the cases; Vancomycin $55 \%$, and was appropriate in $56 \%$ of the cases; Azithromycin $22 \%$, and was appropriate in $53 \%$ of the cases.

The most antibiotic-resistant bacterium was Pseudomonas aeruginosa while the least antibiotic-resistant bacterium was staphylococcus. The highest resistance occurred with Ceftriaxone, Pseudomonas aeruginosa (89\%), Escherichia coli (77\%), Enterobacter (74\%), and Proteus (42\%). Staphylococci sensitivity towards vancomycin was only $3 \%$ (Table 1 ).

The mortality was $19(16 \%), 11(55 \%)$ were due to nosocomial infection and $9(45 \%)$ were due to community infections. The age group associated with the most deaths was the newborns (33\%), followed by the infants $(18 \%)$, late childhood (17\%), and early childhood (11\%). Respiratory tract infections were the leading cause of death (59\%), followed by urinary tract infections $(22 \%)$, blood $11 \%$, and cutaneous $5 \%$; no deaths were associated with meninges. Mortality by pathogen: Enterobacter (27\%), Pseudomonas aeruginosa (24\%), Staphylococcus (18\%), Escherichia coli (9\%), Proteus (6\%), Klebsiella (6\%), Serratia (6\%), and gram-positive bacilli (3\%). 


\section{Discussion}

This first study describes the outcomes of children with positive culture results presenting with nosocomial or community-acquired infections.

The percentage of nosocomial infections in our study was $35 \%$, which is very high compared with a Chinese study, $2.56 \%$ in 2019 , and decreased to $1.39 \%$ in $2020 .{ }^{[9]}$ A similar study in Iran reported the incidence of nosocomial infections as $3 \%{ }^{[10]}$

Staphylococcus aureus was the most common in both nosocomial and community-acquired infections. Escherichia coli was the most common in a Chinese study. ${ }^{[11]}$

Staphylococcus was the most cultured bacteria (75\%) from cerebrospinal fluid (CSF). This is likely to be high due to the presence of only 8 CSF-positive bacterial cultures. Staphylococcus was also the most common causative of meningitis in a study conducted in China, the number of few positive CST bacterial cultures reflects the effect of pretreatments with antibiotics before the CSF sample collection. ${ }^{[12]}$

There is an extremely high bacterial resistance to most of the mentioned antibiotics, especially: ceftriaxone, cefepime, cefotaxime, trimethoprim, gentamycin, levofloxacin, piperacillin with tazobactam. Our results are higher in comparison with a study conducted in China.[11]

Shockingly Staphylococcus sensitivity towards vancomycin was only 3\% despite the usage of vancomycin with treating susceptible infections such as methicillin-resistant Staphylococcus aureus. ${ }^{[13]}$

Sadly, the number of deaths was: $19(16 \%)$, nosocomial infection $11(55 \%)$ and $9(45 \%)$ communityacquired infections $9(45 \%)$. Our results are excruciatingly higher compared with nosocomial infections in China $(0.02 \%)$.

We acknowledge that there are some limitations to this study mainly with the retrospective aspect of data collection. Struggles with data collection included: disorganized medical records, subjective aspects, and illegible handwriting.

\section{Conclusion}

There is an extreme increase in bacterial resistance to most antibiotics. Pretreatment with antibiotics seems inappropriate before receiving the culture results due to the low bacterial sensitivity and high resistance. Also, unwanted side effects of antibiotic use, and incurring financial expenses without any benefit. The efficacy of the remaining antibiotics to which the bacteria are sensitive must be maintained through rationalization of its use. Educational programs targeting doctors, pharmacists, and the community will aid rational use of antimicrobials; similar programs have been implemented in various countries. 


\section{Declarations}

\section{Acknowledgments:}

We are thankful to the management of the Syrian Private University and Damascus Hospital for their support in the field of medical training and research. We would also like to thank Dr Fatema Mohsen for her help and support in the paper.

\section{Funding:}

This research received no specific grant from SPU or any other funding agency in the public, commercial or non-profit sectors.

\section{Availability of data and materials:}

All data related to this paper's conclusion are available and stored by the authors. All data are available from the corresponding author on a reasonable request.

\section{Conflict of interest:}

The authors declare that they have no conflict of interest.

\section{Authors' contributions:}

FK and AH conceptualized the study, participated in the design, wrote the study protocol, performed the statistical analysis, participated in data collection, did a literature search, and drafted the manuscript. MA did a literature search and revision of the draft. All authors read and approved the final draft.

\section{Tables}


Table 1. Antibiotic resistance and sensitivity bacteria

\begin{tabular}{|c|c|c|c|c|c|}
\hline \multicolumn{6}{|c|}{ Resistance } \\
\hline & Escherichia coli & Enterobacter & Proteus & Pseudomonas & Staphylococcus \\
\hline Amikacin & $23 \%$ & $36 \%$ & $33 \%$ & $59 \%$ & $27 \%$ \\
\hline Augmentin & $27 \%$ & $28 \%$ & $50 \%$ & $52 \%$ & $12 \%$ \\
\hline Cefuroxime & $14 \%$ & $28 \%$ & $17 \%$ & $56 \%$ & $20 \%$ \\
\hline Ceftazidime & $32 \%$ & $18 \%$ & $22 \%$ & $41 \%$ & $28 \%$ \\
\hline Ceftriaxone & $77 \%$ & $74 \%$ & $72 \%$ & $89 \%$ & $53 \%$ \\
\hline Cefaclor & $32 \%$ & $26 \%$ & $28 \%$ & $48 \%$ & $20 \%$ \\
\hline Cefepime & $50 \%$ & $46 \%$ & $44 \%$ & $63 \%$ & $37 \%$ \\
\hline Cefixime & $45 \%$ & $46 \%$ & $61 \%$ & $19 \%$ & $28 \%$ \\
\hline Cefotaxime & $68 \%$ & $59 \%$ & $56 \%$ & $67 \%$ & $53 \%$ \\
\hline Trimethoprim & $82 \%$ & $77 \%$ & $61 \%$ & $59 \%$ & $53 \%$ \\
\hline Cefpodoxime & $32 \%$ & $36 \%$ & $50 \%$ & $37 \%$ & $45 \%$ \\
\hline Cefdinir & $50 \%$ & $41 \%$ & $0 \%$ & $15 \%$ & $20 \%$ \\
\hline Ciprofloxacin & $55 \%$ & $23 \%$ & $28 \%$ & $26 \%$ & $22 \%$ \\
\hline Colistin & $5 \%$ & $3 \%$ & $6 \%$ & $7 \%$ & $15 \%$ \\
\hline Fosfomycin & $14 \%$ & $23 \%$ & $44 \%$ & $22 \%$ & $3 \%$ \\
\hline Gentamicin & $50 \%$ & $41 \%$ & $72 \%$ & $33 \%$ & $33 \%$ \\
\hline Imipenem & $14 \%$ & $26 \%$ & $22 \%$ & $44 \%$ & $12 \%$ \\
\hline
\end{tabular}




\begin{tabular}{|c|c|c|c|c|c|} 
Levofloxacin & $50 \%$ & $31 \%$ & $28 \%$ & $44 \%$ & $37 \%$ \\
\hline piper/tazo & $27 \%$ & $31 \%$ & $44 \%$ & $70 \%$ & $20 \%$ \\
\hline Nalidixic Acid & $41 \%$ & $38 \%$ & $44 \%$ & $15 \%$ & $15 \%$ \\
\hline Norfloxacin & $5 \%$ & $8 \%$ & $0 \%$ & $4 \%$ & $3 \%$ \\
\hline Pefloxacin & $18 \%$ & $5 \%$ & $11 \%$ & $26 \%$ & $13 \%$ \\
\hline Clarithromycin & $5 \%$ & $28 \%$ & $33 \%$ & $44 \%$ & $33 \%$ \\
\hline Vancomycin & - & - & - & - & $27 \%$ \\
\hline
\end{tabular}

Sensitivity

\begin{tabular}{|c|c|c|c|c|c|}
\hline & Escherichia coli & Enterobacter & Proteus & Pseudomonas & Staphylococcus \\
\hline Amikacin & $41 \%$ & $28 \%$ & $17 \%$ & $30 \%$ & $40 \%$ \\
\hline Augmentin & $14 \%$ & $5 \%$ & $0 \%$ & $0 \%$ & $27 \%$ \\
\hline Cefuroxime & $0 \%$ & $0 \%$ & $0 \%$ & $0 \%$ & $5 \%$ \\
\hline Ceftazidime & $5 \%$ & $3 \%$ & $0 \%$ & $4 \%$ & $5 \%$ \\
\hline Ceftriaxone & $5 \%$ & $0 \%$ & $6 \%$ & $0 \%$ & $25 \%$ \\
\hline Cefaclor & $0 \%$ & $0 \%$ & $0 \%$ & $0 \%$ & $17 \%$ \\
\hline Cefepime & $9 \%$ & $0 \%$ & $0 \%$ & $11 \%$ & $15 \%$ \\
\hline Cefixime & $0 \%$ & $5 \%$ & $0 \%$ & $4 \%$ & $7 \%$ \\
\hline Cefotaxime & $5 \%$ & $3 \%$ & $0 \%$ & $0 \%$ & $20 \%$ \\
\hline Trimethoprim & $0 \%$ & $5 \%$ & $6 \%$ & $0 \%$ & $17 \%$ \\
\hline Cefpodoxime & $0 \%$ & $0 \%$ & $0 \%$ & $0 \%$ & $2 \%$ \\
\hline Cefdinir & $5 \%$ & $3 \%$ & $6 \%$ & $0 \%$ & $10 \%$ \\
\hline
\end{tabular}




\begin{tabular}{|c|c|c|c|c|c|}
\hline Ciprofloxacin & $5 \%$ & $10 \%$ & $0 \%$ & $4 \%$ & $15 \%$ \\
\hline Colistin & $18 \%$ & $8 \%$ & $0 \%$ & $7 \%$ & $12 \%$ \\
\hline Fosfomycin & $32 \%$ & $18 \%$ & $0 \%$ & $0 \%$ & $15 \%$ \\
\hline Gentamicin & $0 \%$ & $23 \%$ & $11 \%$ & $15 \%$ & $12 \%$ \\
\hline Imipenem & $23 \%$ & $36 \%$ & $17 \%$ & $22 \%$ & $43 \%$ \\
\hline Levofloxacin & $32 \%$ & $31 \%$ & $33 \%$ & $11 \%$ & $28 \%$ \\
\hline piper/tazo & $18 \%$ & $15 \%$ & $17 \%$ & $4 \%$ & $23 \%$ \\
\hline Norfloxacin & $14 \%$ & $0 \%$ & $6 \%$ & $0 \%$ & $0 \%$ \\
\hline Vancomycin & - & - & - & - & $3 \%$ \\
\hline Clarithromycin & $0 \%$ & $0 \%$ & $0 \%$ & $4 \%$ & $0 \%$ \\
\hline
\end{tabular}

\section{References}

1. Murray CJ, Ikuta KS, Sharara F, Swetschinski L, Aguilar GR, Gray A, et al. Global burden of bacterial antimicrobial resistance in 2019: a systematic analysis. The Lancet. 2022. doi: https://doi.org/10.1016/S0140-6736(21)02724-0.

2. Al-Faham Z, Habboub G, Takriti F. The sale of antibiotics without prescription in pharmacies in Damascus, Syria. The Journal of Infection in Developing Countries. 2011;5(05):396-9. doi: https://doi.org/10.3855/jidc.1248.

3. Tourmousoglou C, Yiannakopoulou EC, Kalapothaki V, Bramis J, Papadopoulos JS. Adherence to guidelines for antibiotic prophylaxis in general surgery: a critical appraisal. Journal of Antimicrobial Chemotherapy. 2008;61(1):214-8. doi: https://doi.org/10.1093/jac/dkm406.

4. Alhomoud F, Aljamea Z, Almahasnah R, Alkhalifah K, Basalelah L, Alhomoud FK. Self-medication and self-prescription with antibiotics in the Middle East-do they really happen? A systematic review of the prevalence, possible reasons, and outcomes. International journal of infectious diseases. 2017;57:3-12. doi: https://doi.org/10.1016/j.ijid.2017.01.014. 
5. Bahnassi AJJoTUMS. A qualitative analysis of pharmacists' attitudes and practices regarding the sale of antibiotics without prescription in Syria. Journal of Taibah University Medical Sciences. 2015;10(2):227-33. doi: https://doi.org/10.1016/j.jtumed.2014.09.001.

6. Syrian Syndicate for Pharmacists. (Laws and orders that coordinate pharmacy career in Syria). Damascus, Syrian Syndicate for Pharmacists (In Arabic). Damascus: Syrian Syndicate for Pharmacists, Al-Shadi Publisher 1994.

7. Reinheimer C, Kempf VA, Göttig S, Hogardt M, Wichelhaus TA, O'Rourke F, et al. Multidrug-resistant organisms detected in refugee patients admitted to a University Hospital, Germany June-December 2015. Eurosurveillance. 2016;21(2):30110. doi: http://dx.doi.org/10.2807/1560-

7917.ES.2016.21.2.30110.

8. Jakovljevic M, Jurisevic M, Mouselli S. Antibiotic resistance in Syria: a local problem turns into a global threat. Frontiers in public health. 2018;6:212. doi: https://doi.org/10.3389/fpubh.2018.00212.

9. Su C, Zhang Z, Zhao X, Peng H, Hong Y, Huang L, et al. Changes in prevalence of nosocomial infection pre- and post-COVID-19 pandemic from a tertiary Hospital in China. BMC infectious diseases. 2021;21(1):693. doi: 10.1186/s12879-021-06396-x.

10. Pezhman B, Fatemeh R, Amir R, Mahboobeh R, Mohammad F. Nosocomial infections in an Iranian educational hospital: an evaluation study of the Iranian nosocomial infection surveillance system. BMC infectious diseases. 2021;21(1):1256. doi: 10.1186/s12879-021-06948-1.

11. Fu P, Xu H, Jing C, Deng J, Wang H, Hua C, et al. Bacterial Epidemiology and Antimicrobial Resistance Profiles in Children Reported by the ISPED Program in China, 2016 to 2020. Microbiology spectrum. 2021;9(3):e0028321. doi: 10.1128/Spectrum.00283-21.

12. Peng X, Zhu Q, Liu J, Zeng M, Qiu Y, Zhu C, et al. Prevalence and antimicrobial resistance patterns of bacteria isolated from cerebrospinal fluid among children with bacterial meningitis in China from 2016 to 2018: a multicenter retrospective study. Antimicrobial resistance and infection control. 2021;10(1):24. doi: 10.1186/s13756-021-00895-x.

13. Bradley JS. Nelson's pediatric antimicrobial therapy. American Academy of Pediatrics Grove Village; 2012.

\section{Abbreviations}

MDR: Multidrug-Resistant, CSF: Cerebrospinal Fluid.

\section{Figures}




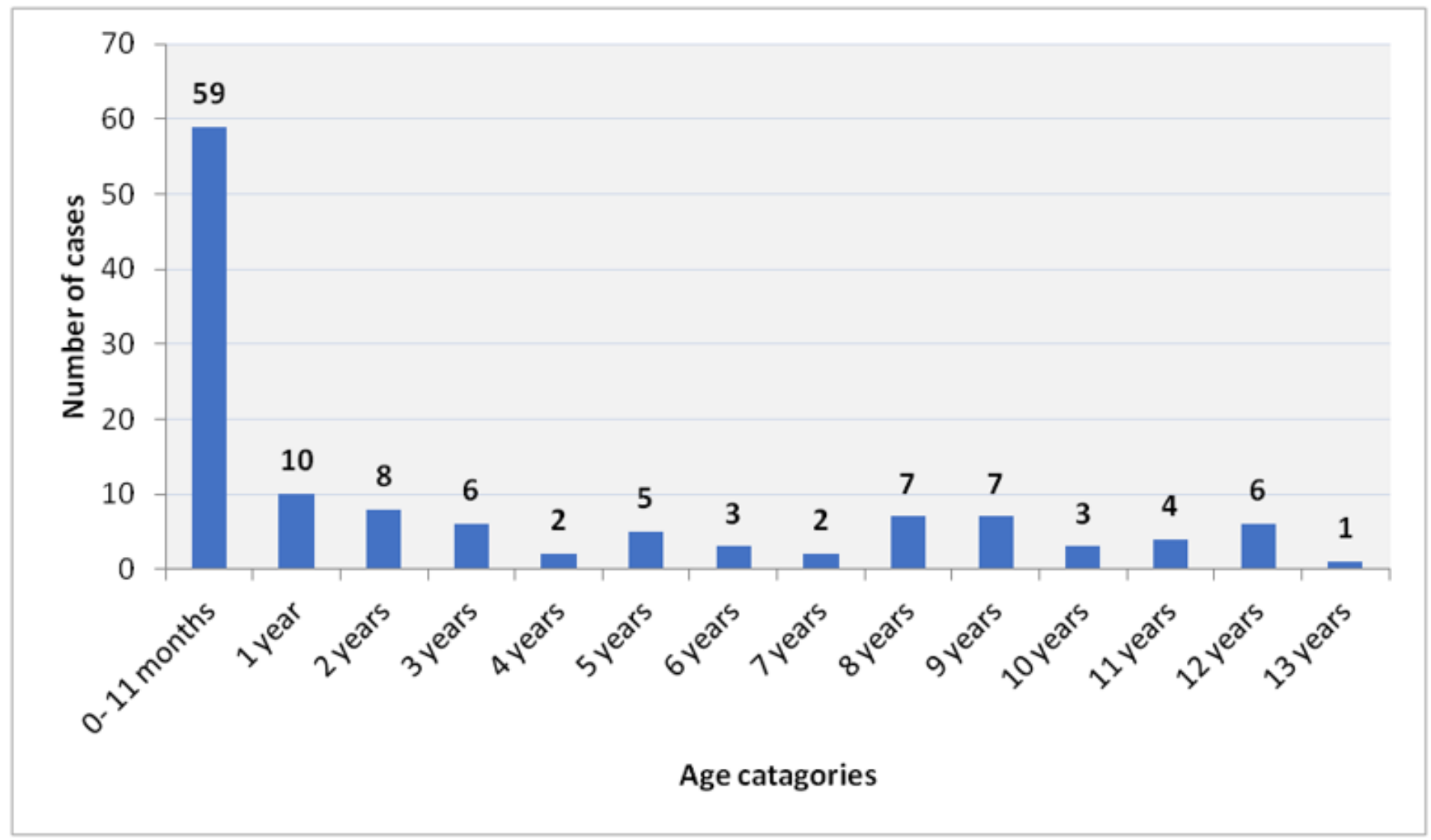

Figure 1

Positive culture results by age incidence 